\title{
La navigation professionnelle: une étude comparative de deux modèles canadiens
}

par Lise Fillion, Sandra Cook, Anne-Marie Veillette, Marie de Serres, Michèle Aubin, François Rainville, Margaret Fitch et Richard Doll

\section{Abrégé}

Dans de nombreux programmes de lutte contre le cancer, la navigation en oncologie est devenue une stratégie ciblée visant à améliorer l'accès aux soins de soutien ainsi que l'expérience des patients qui reçoivent des

\section{Au sujet des auteurs}

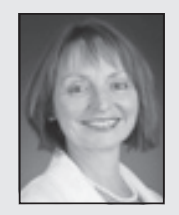

Lise Fillion, inf., Ph.D. (Psychologie), Professeure, Faculté des sciences infirmières, Université Laval, Centre de recherche en cancérologie de l'Université Laval, Hôtel-Dieu de Québec

Toute correspondance se rapportant à cet article doit être adressée à Lise Fillion, Centre de recherche en cancérologie de l'Université Laval, Hôtel-Dieu de Québec, 9 rue McMahon, Québec (Québec), Canada, G1R 2J6. Tél.: 418-525-4444 Poste 15754, Téléc.: 418-691-2920, Lise.Fillion@fsi.ulaval.ca

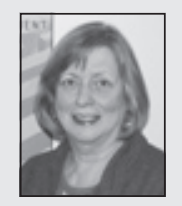

Sandra Cook, inf., B.A., H.S.M., Gestionnaire, Patient Navigation and Surgical Oncology Network, Cancer Care Nova Scotia

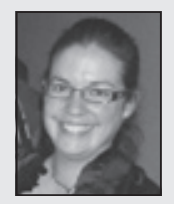

Anne-Marie Veillette, Anth., M.A., Coordonnatrice de la recherche, Centre de recherche en cancérologie de l'Université Laval, Hôtel-Dieu de Québec

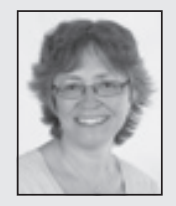

Marie de Serres, inf., M.Sc., Infirmière clinicienne spécialisée, CSIO(C), CHUQ Hôtel-Dieu de Québec

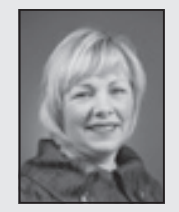

Michèle Aubin, M.D., Ph.D., FCFP, CCMF, Professeure, détentrice de la chaire en soins palliatifs de l'Université Laval, Faculté de médecine, Université Laval, Unité de médecine familiale-Laval, CSSS Vieille-Capitale

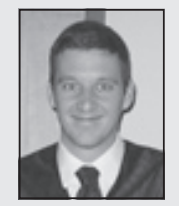

François Rainville, M.S.S., Travailleur social, CHUQ Centre de recherche en cancérologie de l'Université Laval, Hôtel-Dieu de Québec

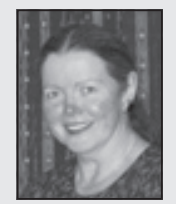

Margaret Fitch, inf., M.Sc.inf., Ph.D., Chef, Soins infirmiers en oncologie, Codirectrice, Programme de soutien au patient et à la famille, Centre de cancérologie Odette, Centre des sciences de la santé Sunnybrook

Richard Doll, M.S.S., M.Sc., Directeur, Centre de recherche socio-comportementale, et Responsable provincial, Réadaptation oncologique, BC Cancer Agency soins en cancérologie. Cette étude vise à améliorer la compréhension de la navigation professionnelle par la comparaison de deux modèles canadiens du rôle associé à la navigation professionnelle en oncologie: l'infirmière pivot en oncologie (IPO) du Québec et l'infirmière responsable de la navigation professionnelle en oncologie (Cancer patient navigator$C P N)$ de la Nouvelle-Écosse. Nous avons mené des entrevues qualitatives auprès de d'IPO et de CPN, de patients et de membres de leur famille, de membres du personnel de première ligne, de médecins et d'administrateurs des soins de santé (entrevues: $n=49$; groupes de discussion: $n=10$ ). Les deux modèles ont été analysés au moyen du cadre conceptuel sur la navigation professionnelle (Fillion et al., 2012). Bien que les modèles soient différents, les résultats indiquent que les Intervenants pivots (IP) en oncologie des deux programmes ont des fonctions semblables et font face à des défis similaires. Cette étude met en relief la complexité et la valeur de la navigation en oncologie et recommande des mesures pertinentes qui visent à optimiser sa gestion au sein du système de soins.

À mesure que les traitements et les approches liés au cancer se complexifient, les patients et leur famille doivent apprendre à naviguer dans un système de santé fragmenté. Dans de nombreux pays, les programmes de lutte contre le cancer reconnaissent le besoin d'améliorer la continuité des soins et la qualité de vie des patients. Les programmes de navigation en oncologie ont vu le jour comme stratégie ciblée visant à améliorer l'expérience des patients qui reçoivent des soins en oncologie. (Doll et al., 2007; Fitch, 2008; Haggerty et al., 2003). Au Canada, le Partenariat canadien contre le cancer (PCCC) appuie l'adoption de la navigation en oncologie en tant qu'élément crucial de l'amélioration des soins cliniques et des soins de soutien en cancérologie. Le PCCC propose trois modes ou types de navigation en oncologie: l'autonavigation (ou navigation virtuelle), effectuée par le biais d'aides virtuelles ou en ligne; la navigation non professionnelle, menée par des bénévoles ou des pairs ayant survécu au cancer; et la navigation professionnelle, réalisée par des intervenants pivots rémunérés. Les trois types comportent différentes responsabilités, attentes envers le rôle et résultats escomptés (PCCC, 2010). En vue de mieux comprendre un de ces modes, la navigation professionnelle, nous comparons dans cette étude deux programmes bien établis au Canada.

\section{La navigation professionnelle en oncologie}

Certaines études empiriques ont déjà examiné la mise en œuvre de différents modèles de navigation professionnelle en oncologie au Canada (Cancer Care Nova Scotia, 2004; Fillion et al., 2006; Plante \& Joannette, 2009a, 2009b; Skrutkowski et al., 2008; Tremblay, 2008). Parker et ses collègues (2010) ont noté qu'à ce jour, la recherche a proposé plusieurs façons de décrire et définir le rôle associé à la navigation professionnelle, mais qu'il y a peu de consensus. Dans leurs travaux, Thorne et Truant (2010) ont décrit la navigation professionnelle comme une solution symbolique à des problèmes créés par la fragmentation et le manque d'intégration du système de soins en oncologie. D'autres auteurs ont décrit les professionnels assumant ce rôle comme des gestionnaires de cas dans les milieux de soins liés au cancer (Lantz, Keeton, Romano \& Degroff, 2004). Cependant, bien que la gestion de cas puisse contribuer à améliorer les délais et la continuité des approches dans le continuum des soins en cancérologie, elle ne rend pas nécessairement l'expérience globale du cancer congruente et cohérente du point de vue du patient. On constate encore un manque de soins de soutien centrés sur la personne (Fitch, 2008).

$\mathrm{Au}$ Canada, la navigation professionnelle correspond généralement à un modèle médical ou social plus englobant de gestion de cas visant à humaniser la trajectoire des soins et à favoriser 
l'empowerment du patient (Fillion et al., 2006). Ce rôle est en fait plutôt complexe, puisqu'il comprend des fonctions organisationnelles et des fonctions cliniques (Doll et al., 2007).

Fillion et al. (2012) reconnaissent cette structure bidimensionnelle dans la définition de la navigation professionnelle qu'ils proposent: la première dimension contribue à l'amélioration de la continuité des soins de santé, tandis que la seconde vise à favoriser l'empowerment des patients et de leur famille dans la lutte contre le cancer. La première dimension correspond à la "faciliation de la continuité des soins". Fillion et ses collaborateurs (2012) ont adapté le modèle théorique de continuité des soins-tel que proposé par Haggerty (2003) pour le contexte de l'oncologie-de façon à inclure trois concepts, à savoir la continuité informationnelle, la continuité des approches thérapeutiques et la continuité relationnelle. La deuxième dimension, axée sur la promotion de l'empowerment des patients et de leur famille, intègre trois concepts différents, soit la capacité de coping actif (Carver, Scheier \& Weintraub, 1989), l'auto-prise en charge du cancer (Bulsara, Styles, Ward \& Bulsara, 2006) et les soins de soutien (Fitch, 2008) (pour plus de détails, voir Fillion et al., 2012).

Lors du processus de validation, le contenu du cadre de navigation bidimensionnelle a fait l'objet d'un consensus parmi les IP en oncologie, les spécialistes, les gestionnaires, les cliniciens et les patients atteints d'un cancer ainsi que leurs êtres chers, et ce, d'un bout à l'autre du Canada (Fillion et al., 2012). Le cadre bidimensionnel comprend une gamme de fonctions organisationnelles et cliniques liées respectivement à chacun des concepts décrits, que peu de professionnels autres les infirmières en oncologie et les travailleurs sociaux bien formés en oncologie psychosociale sont en mesure de réaliser.

Deux programmes provinciaux de navigation professionnelle en oncologie sont apparus au début des années 2000: l'infimière pivot en oncologie (IPO) au Québec (De Serres \& Beauchesne, 2000) et l'infirmière responsable de navigation professessionnelle (Cancer patient navigator-CPN) en Nouvelle Écosse en Nouvelle-Écosse (Cancer Care Nova Scotia, 2004). Les deux mettent à contribution des infirmières spécialisées en oncologie-ayant complété une formation professionnelle reconnue-dans le rôle d'IP en oncologie. Elles travaillent auprès des patients qui viennent de recevoir un diagnostic de cancer, des personnes qui reçoivent actuellement un traitement ou qui sont en période de suivi et des survivants du cancer. Les IPO et les CPN aident les patients, leur famille et les professionnels de la santé à s'y retrouver dans le labyrinthe d'options et de services offerts pour générer la meilleure qualité de vie et les meilleurs résultats possibles, et ce, à chaque étape de l'expérience du cancer (voir le modèle de Fillion et al. (2012) pour des exemples concrets des fonctions clés).

Pour l'instant, les différences entre les deux modèles demeurent peu documentées. Dans leur description de leur cadre bidimensionnel, Fillion et ses collaborateurs (2012) ont démontré que les IPO et les CPN ont des rôles très semblables. Les différences sur la façon d'exécuter les fonctions n'ont toutefois pas été explicitées. Cette étude vise à contribuer à une meilleure compréhension de la navigation en oncologie en comparant ces deux modèles de navigation professionnelle.

\section{Méthodologie \\ Démarche de recherche}

Pour mieux comprendre la navigation en oncologie, cette étude qualitative descriptive a employé un devis à cas multiples (Yin, 1994) mettant en jeu deux cas: 1) l'infirmière pivot en oncologie (IPO) au Québec et 2) l'intervenant pivot en oncologie (CPN) en NouvelleÉcosse. L'objectif de l'étude était de comparer les IPO et les CPN et de déterminer si les deux rôles présentaient des différences. Les chercheurs ont utilisé un processus d'évaluation ciblée (Fillion et al., 2011; Patton, 1997) ainsi que le modèle conceptuel bidimensionnel de Fillion et al. (2012) à titre de référence (voir tableau 1).

\section{Participants et recrutement}

L'échantillon intentionnel comprenait huit groupes de participants : 1) des IPO; 2) des CPN; 3) des médecins spécialistes en oncologie; 4) des infirmières et du personnel en oncologie; 5) des administrateurs; 6) des médecins de famille; 7) des patients atteints d'un cancer; 8) des membres des familles et de l'entourage. Les groupes 1, 3, 4, 5, 6 et 8 participaient au Québec, et les groupes 2 et 5 participaient en Nouvelle-Écosse. Les chercheurs ont utilisé un échantillonnage non aléatoire axé sur l'information afin de recueillir les opinions et les expériences approfondies des participants.

\begin{tabular}{|c|c|}
\hline Dimensions & oncepts \\
\hline $\begin{array}{c}\text { Facilitation de la } \\
\text { continuité des soins } \\
\text { (Les soins sont perçus comme } \\
\text { cohérents et interconnectés) } \\
\text { Fonctions organisationnelles } \\
\text { du rôle } \\
\text { (Dimension axée sur } \\
\text { le système de santé) }\end{array}$ & $\begin{array}{l}\text { Continuité informationnelle } \\
\text { Utilisation de l'information axée sur la maladie ou sur la personne afin de rendre les soins actuels plus } \\
\text { pertinents pour chaque individu. L'information permet d'établir des liens entre les soins prodigués } \\
\text { par différents fournisseurs et entre différents évènements de santé (Haggerty et al., 2003). } \\
\text { Continuité des approches thérapeutiques } \\
\text { Une approche cohérente et congruente en matière de gestion du cancer qui répond aux besoins } \\
\text { changeants des patients. Confère un sentiment de prévisibilité et de sécurité par rapport aux soins } \\
\text { futurs, à la fois pour les patients et pour les fournisseurs. } \\
\text { Continuité relationnelle } \\
\text { Une relation thérapeutique entre un patient et au moins un fournisseur de soins qui acquiert une } \\
\text { connaissance croissante du patient en tant que personne et qui établit un lien entre les soins passés, } \\
\text { présents et futurs (Haggerty et al., 2003). }\end{array}$ \\
\hline $\begin{array}{l}\text { Promotion de l'empowerment } \\
\text { des patients et de leur famille } \\
\text { (fournisseurs de soins en tant } \\
\text { que partenaires encourageants) }\end{array}$ & $\begin{array}{l}\text { Coping actif } \\
\text { Mesures actives qui visent à enrayer ou à éviter les facteurs de stress ou du moins à en réduire les } \\
\text { effets (Carver et al., 1989). } \\
\text { Auto-prise en charge du cancer } \\
\text { Appuyer la personne et sa famille et renforcer sa capacité d'accepter la maladie et de reprendre le } \\
\text { contrôle, quel que soit le pronostic (Bulsara et al., 2006). }\end{array}$ \\
\hline $\begin{array}{l}\text { Fonctions cliniques du rôle } \\
\text { (Dimension axée sur le patient) }\end{array}$ & $\begin{array}{l}\text { Soins de soutien } \\
\text { Fournir les services nécessaires tels que définis par les personnes atteintes ou touchées par un cancer } \\
\text { afin de répondre à leurs besoins physiques, informationnels, pratiques, émotionnels, psychologiques, } \\
\text { sociaux et spirituels (Fitch, 2008). }\end{array}$ \\
\hline
\end{tabular}


Les IPO, les CPN et les administrateurs de la Nouvelle-Écosse et du Québec ont contribué au recrutement en préparant des listes de participants admissibles que l'équipe de recherche a contactés par téléphone. Les patients ont également contribué au processus de recrutement en invitant les membres de leur famille à participer. Tous les participants intéressés ont été contactés par téléphone par un membre de l'équipe de recherche, et ils ont tous signé un formulaire de consentement. Le projet de recherche a reçu l'approbation du comité d'éthique de la recherche de chaque établissement participant.

\section{Collecte de données}

Les chercheurs ont réalisé une collecte de données multimodale : 1) documents (recension de la documentation scientifique, outils et ressources des IPO/CPN); 2) entrevues individuelles $(n=49)$; et 3) groupes de discussion $(n=10)$. Pour les guides des entrevues individuelles et des discussions de groupe (voir annexes 1 et 2 pour des exemples), les questions étaient formulées d'après le cadre bidimensionnel de navigation professionnelle élaboré par Fillion et ses collaborateurs (2012) et selon un processus d'évaluation ciblé (Fillion et al., 2011; Patton, 1997).

\section{Analyse des données}

Toutes les entrevues individuelles et les discussions de groupe ont fait l'objet d'un enregistrement sonore. Les enregistrements ont ensuite été entièrement transcrits, et les transcriptions ont été étudiées. Afin de protéger la confidentialité des participants, aucune donnée-nominale ou autre-susceptible d'identifier les participants n'a été transcrite. Les chercheurs ont identifié chacune des transcriptions au moyen d'un numéro et les ont rangées par groupe (de un à huit) et par province (Québec, Nouvelle-Écosse).

Les transcriptions ont été classées à la fois à l'aide du cadre bidimensionnel de navigation professionnelle et du processus d'évaluation ciblée. En effet, afin d'englober la diversité des fonctions des IP en oncologie, l'analyse des données a suivi les deux dimensions du cadre de navigation professionnelle (Fillion et al., 2012). Le programme NVivo $7^{\circ}$ a permis aux chercheurs de créer et d'attribuer un code descriptif à chaque unité de sens (mots, phrases ou paragraphes) qui reflétait un enjeu. Le regroupement de codes semblables a permis de créer des catégories thématiques représentant un ensemble d'éléments conceptuels. Les chercheurs ont effectué l'analyse au moyen de l'approche de Strauss et Corbin (1990). L'équipe de recherche s'est livrée à un processus constant-comparatif d'analyse des données, et ce, de façon continue, afin d'assurer la saturation des données et d'optimiser la validité de l'analyse. Une analyse des données en fonction des provinces et au moyen d'un processus de comparaison inductif et déductif a permis de dégager des similitudes et des différences entre les deux modèles de navigation professionnelle et a contribué à améliorer notre compréhension de la navigation en oncologie.

\section{Résultats}

$\mathrm{Au}$ total, 100 personnes ont pris volontairement part à cette étude : le groupe d'IPO $(n=21)$ et le groupe de CPN $(n=5)$; le groupe de médecins spécialistes en oncologie (un(e) spécialiste du poumon, un(e) oncologue, un(e) urologue, un(e) radio-oncologue, un(e) psychiatre) $(n=5)$; le groupe des médecins de famille $(n=11)$; le groupe des autres travailleurs en oncologie interagissant avec les IPO (infirmières, travailleurs sociaux, travailleurs et coordonnateurs en ressources communautaires, psychologue, ergothérapeute, physiothérapeute, nutritionniste) $(n=27)$; le groupe de gestionnaires et d'administrateurs qui interagissait avec les IPO du Québec $(n=8)$ et avec les CPN de la Nouvelle-Écosse $(n=5)$; et le groupe de patients en oncologie ayant reçu des services d'IPO $(n=13)$ et leurs êtres chers $(n=5)$ (voir les tableaux 2 et 3 pour plus de détails).

\begin{tabular}{|c|c|c|c|c|c|c|c|}
\hline & $\begin{array}{l}\text { CPN de } \\
\text { Nouvelle- } \\
\text { Écosse }(n=5)\end{array}$ & $\begin{array}{l}\text { Gestionnaires } \\
\text { de Nouvelle- } \\
\text { Écosse }(n=5)\end{array}$ & $\begin{array}{l}\text { IPO du } \\
\text { Québec } \\
(n=21)\end{array}$ & $\begin{array}{l}\text { Gestionnaires } \\
\text { du Québec } \\
(\mathrm{n}=8)\end{array}$ & $\begin{array}{l}\text { Médecins } \\
\text { spécialistes } \\
(n=5)\end{array}$ & $\begin{array}{l}\text { Médecins } \\
\text { de famille } \\
(\mathrm{n}=11)\end{array}$ & $\begin{array}{l}\text { Autres } \\
\text { travailleurs } \\
\text { en oncologie } \\
(n=27)\end{array}$ \\
\hline \multicolumn{8}{|l|}{ Sexe } \\
\hline Femmes & $5(100 \%)$ & $5(100 \%)$ & $21(100 \%)$ & $6(75 \%)$ & $3(60 \%)$ & $5(45 \%)$ & 25 (93\%) \\
\hline Hommes & $0(0 \%)$ & $0(0 \%)$ & $0(0 \%)$ & $2(25 \%)$ & $2(40 \%)$ & $6(55 \%)$ & $2(7 \%)$ \\
\hline Âge, $M(E ́ T)$ & $46,00(9,20)$ & $47,00(10,15)$ & $43,10(9,36)$ & $47,00(6,35)$ & $43,80(10,40)$ & $48,82(6,94)$ & $47,70(8,68)$ \\
\hline \multicolumn{8}{|l|}{ Années d'expérience } \\
\hline Total, $M(E ́ T)$ & $20,25(10,69)$ & $17,75(8,62)$ & $21,81(10,55)$ & $27,75(6,09)$ & $14,90(10,51)$ & $22,73(8,11)$ & $23,15(9,24)$ \\
\hline En oncologie, $M(E ́ T)$ & $6,63(5,96)$ & $9,75(7,85)$ & $10,24(9,96)$ & $15,75(11,13)$ & $15,80(9,31)$ & $18,50(7,87)$ & $9,43(7,81)$ \\
\hline $\begin{array}{l}\text { Dans son poste actuel, } \\
M(E ́ T)\end{array}$ & $4,63(2,63)$ & $7,50(8,96)$ & $2,17(1,97)$ & $2,88(2,10)$ & $13,90(10,62)$ & $\begin{array}{c}20,73 \\
(10,12) \\
\end{array}$ & $8,50(7,36)$ \\
\hline $\begin{array}{l}\text { Estimation du nombre } \\
\text { de nouveaux patients en } \\
\text { oncologie suivis chaque } \\
\text { année, } M(E ́ T)\end{array}$ & $228,33(106,81)$ & $\begin{array}{l}\text { Ne s'applique } \\
\text { pas }\end{array}$ & $\begin{array}{c}161,89 \\
(106,21)\end{array}$ & $\begin{array}{c}\text { Ne s'applique } \\
\text { pas }\end{array}$ & $\begin{array}{c}250,00 \\
(212,13)\end{array}$ & $\begin{array}{c}53,18 \\
(85,12)\end{array}$ & $\begin{array}{c}\text { Ne s'applique } \\
\text { pas }\end{array}$ \\
\hline \multicolumn{8}{|l|}{ Scolarité } \\
\hline $\begin{array}{l}\text { Diplôme en sciences } \\
\text { infirmières (Nouvelle- } \\
\text { Écosse) }\end{array}$ & $2(40 \%)$ & $0(0 \%)$ & $0(0 \%)$ & $0(0 \%)$ & $0(0 \%)$ & $0(0 \%)$ & $0(0 \%)$ \\
\hline Collège (Québec) & $0(0 \%)$ & $0(0 \%)$ & $0(0 \%)$ & $1(12,5 \%)$ & $0(0 \%)$ & $0(0 \%)$ & $8(30 \%)$ \\
\hline Baccalauréat & $3(60 \%)$ & $4(80 \%)$ & $20(95 \%)$ & $1(12,5 \%)$ & $0(0 \%)$ & $0(0 \%)$ & $17(63 \%)$ \\
\hline Maîtrise & $0(0 \%)$ & $1(20 \%)$ & $1(5 \%)$ & $5(62,5 \%)$ & $0(0 \%)$ & $0(0 \%)$ & $2(7 \%)$ \\
\hline Doctorat & $0(0 \%)$ & $0(0 \%)$ & $0(0 \%)$ & $1(12,5 \%)$ & $5(100 \%)$ & $11(100 \%)$ & $0(0 \%)$ \\
\hline
\end{tabular}


Facilitation de la continuité des soins Continuité informationnelle

Selon la plupart des participants, les intervenants pivots (IP) en oncologie des deux provinces partagent des renseignements et des conseils opportuns et personnalisés avec les patients atteints d'un cancer et les autres professionnels des équipes interdisciplinaires. En collaborant étroitement avec eux, les IP contribuent activement à améliorer la continuité informationnelle. Les patients, les différents professionnels et les administrateurs ont tous indiqué que les IP étaient des personnes-ressources clés. Cependant, les IP et les gestionnaires des deux provinces ont exprimé le désir d'améliorer la continuité informationnelle par l'adoption de mécanismes systématiques de transfert et de diverses stratégies comme les discussions de cas, la vidéoconférence et les télésoins. Dans les deux provinces, la nondisponibilité de dossiers électroniques s'est révélée être un obstacle à la continuité informationnelle. À l'heure actuelle, l'information est transmise par des moyens traditionnels (verbalement, notes dans les dossiers médicaux, télécopieur, téléphone), sans mécanismes concertés ou systématiques.

Pour ce qui est de l'évaluation initiale des besoins, les IPO et les $\mathrm{CPN}$ ont accès à un outil d'évaluation normalisé. Les deux groupes ont indiqué que cet instrument est utile, mais limité. Les IPO et les CPN ont signalé qu'il est trop long, qu'il prend trop de temps, qu'il se limite à la première étape du continuum et qu'il n'est pas nécessairement approprié pour tous les patients ayant le cancer. Ils ont également indiqué que le transfert d'information présentait des défis dans les deux provinces, mais qu'il y avait quelques différences. Au Québec, les IPO consignent généralement l'information sur les patients dans des dossiers médicaux hospitaliers, auxquels tous les membres de l'équipe spécialisée ont accès. En Nouvelle-Écosse, on a constaté que les autres professionnels n'ont pas accès aux dossiers des CPN parce que ces dernières les gardent dans leurs bureaux, ce qui constitue un obstacle à la continuité informationnelle entre professionnels de la santé. Bref, toutes les IPO et les CPN contribuaient à la continuité informationnelle. L'évaluation des besoins était une fonction clé, mais elle présentait certaines contraintes liées aux outils.

\section{Continuité des approches thérapeutiques}

Dans les deux modèles, les IP en oncologie facilitent la continuité des approches dans les secteurs suivants: dépistage de la détresse, évaluation des besoins, arrimage de ces besoins avec les services et ressources offerts dans l'établissement de santé et au sein de la communauté. Leurs partenariats et réseaux contribuent à combler de nombreuses lacunes. En ce qui a trait au dépistage de la détresse

Tableau 3: Caractéristiques des patients et de leurs proches

\begin{tabular}{|c|c|}
\hline & $\begin{array}{l}\text { Patients en oncologie et } \\
\text { leurs proches }(n=18)\end{array}$ \\
\hline \multicolumn{2}{|l|}{ Sexe } \\
\hline Femmes & $11(61 \%)$ \\
\hline Hommes & 7 (39\%) \\
\hline Âge, $M(E ́ T)$ & $61,00(12,47)$ \\
\hline \multicolumn{2}{|l|}{ Scolarité } \\
\hline Études secondaires non terminées & $2(11 \%)$ \\
\hline École secondaire & 7 (39\%) \\
\hline Diplôme professionnel (Québec) & $1(6 \%)$ \\
\hline Collège (Québec) & $6(33 \%)$ \\
\hline Baccalauréat & $2(11 \%)$ \\
\hline Maîtrise & $0(0 \%)$ \\
\hline Doctorat & $0(0 \%)$ \\
\hline
\end{tabular}

et à l'évaluation des besoins, les IP ont une fois encore signalé leur besoin d'avoir accès à des outils d'évaluation et de dépistage plus pratiques et complets qui puissent servir dans la totalité du continuum de soins, être transférés à d'autres professionnels et être utilisés pour structurer plus efficacement les soins et les références aux autres professionnels..

Afin d'effectuer le dépistage de la détresse et l'évaluation des besoins le plus tôt possible, les IP, dans les deux cas, doivent recevoir des références précoces de la part des médecins, des infirmières, des travailleurs sociaux, des pharmaciens et d'autres professionnels, ainsi que des patients eux-mêmes ou des membres de leur famille. Il n'y avait pas de processus de référence systématique. Certaines IP devaient repérer les patients, ce qui nuisait à leurs autres responsabilités et activités au cours de la journée. Les deux groupes ont indiqué que la continuité des approches devrait commencer le plus tôt possible dans le continuum du cancer et que les mécanismes de référence vers les IP doivent être améliorés.

En plus du dépistage, les deux groupes ont mentionné les éléments suivants comme étant essentiels à la continuité des approches : la schématisation de la trajectoire du cancer, l'indication des services accessibles tout au long de l'expérience et enfin, l'arrimage des besoins non satisfaits avec les ressources appropriées. Comme elles œuvrent au sein de la collectivité, les CPN sont bien placées pour informer leurs patients au sujet des services communautaires. Cependant, par comparaison avec les IPO, elles étaient moins intégrées dans les équipes d'oncologie structurées. Pour les CPN, la schématisation du continuum de soins ou l'explication du plan de soins était parfois un défi. Pour leur part, les IPO (qui étaient basées dans une équipe suprarégionale et spécialisées par site tumoral), étaient davantage en mesure d'élaborer et de schématiser la trajectoire du cancer. Lors de besoin d'information sur les ressources communautaires, les IPO devaient parfois aiguiller les patients vers l'infirmière de liaison ou vers une travailleuse sociale. Selon l'endroit ou elles œuvrent ou leur appartenance à une équipe d'oncologie, les mécanismes conçus pour apparier les ressources et les besoins non satisfaits étaient différents.

\section{Continuité relationnelle}

Les IP en oncologie mettent en place et entretiennent des relations et demeurent présentes à toutes les étapes de la trajectoire des soins, tant que les patients expriment un besoin de leur soutien. Dans les deux cas, elles étaient disponibles du lundi au vendredi pendant les heures de bureau. Cependant, cette disponibilité était parfois compromise par un volume accru de nouveaux cas, de suivis et de crises inattendues. La question de la charge de travail a amené les CPN en Nouvelle Écosse à réduire les visites à domicile et à redéfinir les critères d'accès. De plus, la plupart d'entre elles ont mentionné que le manque d'espaces favorisant l'intimité constituait une barrière à la continuité relationnelle.

Certaines différences ont été notées en matière de continuité relationnelle. Les IPO sont plus actives que les CPN au cours de la phase de traitement. Le rôle des CPN commence souvent plus tôt dans la communauté et autour de la phase diagnostique, et ce rôle devient plus important à la fin de la phase de traitement, lorsque de transition vers la phase de survie.

La continuité relationnelle débute par l'établissement d'une relation thérapeutique. Même si l'implication des IP peut varier en fonction de leur emplacement géographique, les IPO et les CPN demeuraient disponibles tout au long de la trajectoire du cancer. Les deux groupes s'impliquaient activement vers le début et vers la fin des traitements, deux transitions importantes dans le continuum du cancer.

\section{Promotion de l'empowerment des patients et de leur famille Le coping actif}

Il n'y avait pas de grandes différences entre les deux groupes en ce qui concerne ce volet du rôle d'IP en oncologie. Les participantes 
ont expliqué qu'elles soutenaient leurs patients en oncologie dans leur recherche d'information, de soutien et pour l'obtention des références dont ils avaient besoin, et à ainsi satisfaire leurs besoins pratiques et sociaux. Elles contribuaient à l'empowerment des patients en les orientant vers les ressources appropriées, sans toutefois faire le travail pour eux. Plusieurs patients et proches ont confirmé que ces intervenants pivots les avaient aidés à accomplir des tâches ou des activités par eux-mêmes, à résoudre des problèmes ou à gérer leur détresse (p. ex. cerner le problème, explorer des solutions, faciliter le choix d'une solution, aider le patient à la mettre en œuvre et à en évaluer la pertinence). Les IP offrent des renseignements qui aident les patients à prendre des décisions éclairées concernant des changements relatifs au mode de vie. Elles aident les patients et les membres de leur famille à prendre des décisions lors de transitions dans la trajectoire du cancer, en particulier la transition vers les soins palliatifs.

Les IP ont indiqué que les interventions psychosociales constituent des fonctions clés de leur rôle. Dans les deux cas, cependant, elles ont signalé plusieurs défis. Elles ont fait valoir qu'elles auraient besoin de formations ciblées dans les trois domaines suivants: 1) comment motiver les patients par rapport aux changements comportementaux; 2) comment promouvoir les autosoins; 3) quelles interventions poser relativement aux enjeux sexuels et spirituels.

\section{Auto-prise en charge du cancer}

Les deux groupes participaient activement à la facilitation de la gestion du cancer. Elles favorisent une meilleure auto-prise en charge, notamment en évaluant ou en surveillant les symptômes, en effectuant ou en facilitant la gestion des symptômes et en aidant les patients à s'adapter à leur état de santé altéré et à leurs symptômes, de façon proactive et non réactive, par le biais de renseignements opportuns et personnalisés et d'instructions sur les autosoins. Elles jouent également un rôle de porte-parole, puisqu'elles aident les patients et les familles à négocier les soins qu'ils reçoivent. Grâce à l'assistance des IP, les patients se sentent plus en mesure de gérer leur cancer et la trajectoire des soins, IP elles mêmes. De par leur implication accrue pendant les phases de traitement, les IPO participaient encore plus activement à la gestion des symptômes.

\section{Soins de soutien}

Les IP des deux groupes ont fait valoir que leurs fonctions, de dépistage de la détresse, d'évaluation, de soins directs ou d'interventions et de références avaient pour effet d'améliorer l'accès aux soins de soutien pour les patients en oncologie et leur famille. Plus précisément, les deux groupes ont indiqué que le dépistage de la détresse et l'évaluation globale des besoins et des ressources en matière de soins de soutien étaient des fonctions clés. L'évaluation orientait les IP sur le soutien à fournir aux patients et aux proches afin qu'ils cernent et abordent plus efficacement leurs besoins non satisfaits.

\section{Discussion}

Cette étude visait à comparer deux types d'intervenants pivots en oncologie bien établis au Québec et en Nouvelle-Écosse.

Les données ont révélé certaines différences principalement liées aux contextes et aux équipes dans lesquels les intervenantes œuvrent. En effet, les IPO du Québec sont intégrées à des équipes d'oncologie et souvent basées dans un hôpital de soins tertiaires. En revanche, la majorité des CPN de la Nouvelle-Écosse ne sont pas intégrées à des équipes spécialisées en oncologie et oeuvrent en secteur communautaire. Ces deux différences semblent expliquer la majorité des divergences de rôle observées.

Plusieurs similitudes ont aussi été notées, notamment le fait que dans les deux cas, les intervenants pivots étaient des infirmières en oncologie autorisées et qu'elles étaient disponibles tout au long de la trajectoire des soins. Comme le révèlent des études antérieures, le champ de pratique des IP diffère de celui des autres professionnels en oncologie et des infirmières spécialisées en oncologie (Cook et al., 2010). Notamment, les IP peuvent se voir impliquées à toutes les phases de la trajectoire du cancer, et n'exercent pas seulement dans un secteur particulier de la pratique en oncologie comme la chirurgie, la radiothérapie ou la chimiothérapie. Elles jouent un rôle crucial dans la facilitation des transitions au cours du continuum de soins. De plus, elles assurent la continuité relationnelle tout au long de l'expérience du cancer en étant présentes pour les patients et leurs proches à partir du moment du diagnostic jusqu'à l'étape des soins de fin de vie, en passant par les phases de traitement et les récidives (Cancer Care Nova Scotia, 2004; De Serres \& Beauchesne, 2000; Fillion et al., 2012; Tremblay, 2008). De plus, les résultats indiquent que dans les deux provinces, les IP font face à des défis semblables dans l'exécution de leur rôle, à savoir, la facilitation de la continuité des soins et la promotion de l'empowerment des patients et de leur famille.

\section{Continuité des soins}

Dans cette étude, les deux types d'IP ont décrit leur rôle sur le plan de la continuité informationnelle. L'évaluation des besoins s'est révélée être une fonction clé, et elle était associée à des contraintes au niveau des outils qui seront abordées plus loin. Les participants ont indiqué que le transfert de l'information présentait lui aussi des défis, en particulier lorsque les conclusions de l'évaluation n'étaient pas versées dans les dossiers médicaux. Il était clair qu’il fallait rehausser l'efficacité du transfert de l'information dans les dossiers des NPO (manque d'outils de communication normalisés, p. ex. dossiers électroniques). La continuité de l'information était particulièrement compromise pendant la période de suivi, après la fin des traitements. Afin de remédier à la situation, on prône l'adoption de plans de soins de suivi pour les survivants (Earle, 2006; Institute of Medicine [IOM], 2006).

Les résultats révèlent en outre que les IPO et les CPN contribuaient activement à la continuité des approches. Les deux groupes ont mentionné les activités suivantes comme faisant partie de leurs fonctions clés: satisfaire les besoins d'information, schématiser la trajectoire du cancer et apparier les besoins non satisfaits aux ressources appropriées (Bowman \& Grim, 2008; Wilcox \& Bruce, 2010). Il y avait des différences au niveau de l'agencement des mécanismes d'arrimage utilisés, mais les intervenantes du Québec et de la Nouvelle-Écosse faisaient face à des défis semblables. Elles devaient contribuer à la mise en place une coordination cohérente et opportune des services à une période où les plans de soins interdisciplinaires et les partenariats communautaires formels (approches de soins partagés), deux stratégies recommandées par l'IOM (2006), n'existaient pas encore.

Dans les deux cas, les IP contribuaient activement à l'amélioration de la collaboration interprofessionnelle. À ces fins, elles devaient d'abord promouvoir leur rôle, qui constitue en outre une pratique émergente dans le domaine de l'oncologie. L'importance de la continuité des soins dans la navigation professionnelle, ainsi que les défis qui s'y rattachent, a déjà fait l'objet de discussion dans les écrits (PCCC, 2010; Doll et al, 2007; Fillion et al., 2012; Plante \& Joannette, 2009b; Tremblay, 2008). Il est donc recommandé de préciser les zones de chevauchement perçus par les différents professionnels qui travaillent avec les IP. Il est également conseillé de promouvoir et clarifier ce rôle clé auprès des patients et des équipes interprofessionnelles.

Finalement, dans les deux cas, les résultats illustrent l'importance de la navigation professionnelle pour la continuité relationnelle, qui commence par l'établissement d'une relation thérapeutique. Même si le degré d'implication des IP varie en fonction de leur emplacement géographique, les deux groupes (IPO et CPN) demeurent disponibles tout au long de la trajectoire du cancer (PCCC, 2010; De Serres \& Beauchesne, 2000; Fillion et al., 2012; Haggerty et al., 2003; Wilcox \& 
Bruce, 2010). Les deux groupes interviennent activementvers le début et à la fin des traitements, deux transitions importantes dans le continuum du cancer. Le soutien dispensé par les IP pendant ces deux périodes contribuerait par ailleurs à la continuité relationnelle.

\section{Empowerment des patients et de leur famille}

Pour ce qui est de l'empowerment, nos résultats indiquent que les IP ont effectué des interventions psychosociales en vue de renforcer des stratégies de coping actif. Les interventions psychosociales constituent des éléments majeurs pour aider les patients et leur famille à s'adapter à une gamme de changements de vie liés au cancer. Les IP en oncologie ont toutefois indiqué que leur expertise dans certains volets précis de ce domaine présentait parfois des lacunes. Elles auraient avantage à recevoir de la formation en oncologie psychosociale (PCCC, 2010; Tremblay, 2008).

Les résultats ont également révélé que les deux types de navigation professionnelle auraient des effets positifs semblables sur la gestion du cancer. Les IPO et les CPN contribuent à ce résultat en aidant les patients à s'adapter à leur état de santé altéré et à leurs symptômes et à les prendre en charge de façon proactive, et non réactive, par des renseignements opportuns et des instructions personnalisées sur les autosoins (Fitch, 2008). En effet, avec l'aide des IP, la compréhension des patients s'améliore, et leur anxiété diminue. De plus, les patients acquièrent des outils leur permettant de composer avec leurs difficultés, et ils se sentent plus en mesure de se concentrer sur leur rétablissement et sur la reprise en main de leur vie (Wilcox \& Bruce, 2010).

Les résultats ont également illustré certaines différences entre les deux groupes sur le plan de la gestion des symptômes. Les IPO étaient plus actives durant les traitements. Comme elles étaient basées, pour la plupart, dans des milieux hospitaliers, elles étaient en mesure de s'occuper plus directement des symptômes des patients. En Nouvelle-Écosse, les patients qui reçoivent des traitements dans les centres de cancérologie sont assignés à des infirmières en oncologie, qui s'occupent de la majeure partie de la gestion des symptômes. La différence observée entre les deux groupes peut donc s'expliquer par des facteurs liés à l'organisation des soins plutôt que par une réelle différence en matière de rôle.

Finalement, pour ce qui est de la facilitation de l'accès aux soins de soutien, les résultats soulignent l'importance du dépistage de la détresse et de l'évaluation globale des besoins et ressources en soins de soutien. Dans les deux régions géographiques, les intervenantes ont présenté ces activités d'évaluation, qui combinaient des outils et des entrevues narratives, comme des fonctions clés des soins de soutien. Les recensions de la documentation scientifique décrivent elles aussi des outils ou des façons de combiner le questionnaire et l'entrevue clinique en vue de mieux cibler des objectifs cliniques (Wen \& Gustafson, 2004; Richardson, Sitzia, Brown, Medina \& Richardson, 2005).

\section{Outils et formation}

Tous les défis décrits ci-dessus révèlent la nécessité d'améliorer les outils et la formation dans chaque modèle, et ce, pour les deux dimensions du rôle. En effet, au moment de la collecte de données, les IP des deux provinces cherchaient des outils pratiques qui pourraient servir à toutes les étapes du continuum de soins, être transférés à d'autres professionnels et être utilisés pour structurer les soins et les références de façon plus efficace. Les efforts actuels visant à mettre en place des outils systématiques de dépistage de la détresse parmi les IP devraient contribuer à répondre à ce besoin (PCCC, 2009). La question de l'implantation d'un outil systématique de dépistage de la détresse parmi les IP du Québec et de la Nouvelle-Écosse a d'ailleurs été examinée dans un récent article (Fillion et al., 2011). Bref, il est recommandé de poursuivre les efforts de soutien au rôle et aux fonctions des IP (p. ex. des outils informatisés facilitant la coordination des soins, des procédures officielles facilitant la continuité informationnelle, des procédures de références autour du moment du diagnostic, des outils de dépistage de la détresse, des outils d'évaluation complets, ciblés, concis et fonctionnels pouvant servir tout au long du continuum, des indicateurs de l'incidence économique de la navigation professionnelle sur le système de santé, des stratégies permettant de réduire les tâches administratives des IP).

Sur le plan de la formation, des efforts sont réalisés dans les deux provinces pour que toutes les IP reçoivent des formations adéquates. Au Québec, toutes les IPO suivent le programme de formation de la Direction québécoise en cancérologie (DLCC, 2007) et participent à des formations continues. En Nouvelle-Écosse, le programme de navigation en oncologie permet aux CPN d'accéder à l'éducation continue selon leurs besoins. Par exemple, lorsque les CPN ont dû élargir leur champ de compétences dans les soins psychosociaux, des interventions éducatives ont été planifiées en vue d'appuyer cet élargissement. Les résultats indiquent que la formation de base et les efforts investis dans la formation continue à l'intention des IP doivent se poursuivre afin de leur permettre d'assumer pleinement leur rôle, notamment en ce qui a trait aux soins psychosociaux.

\section{Limites}

La première limite correspond au fait que la plupart des participants étaient favorables au rôle de navigation professionnelle. De plus, le nombre d'échantillonages différait d'une province à l'autre et pouvait limiter la comparaison. Malgré ces limites, l'utilisation du cadre conceptuel retenu s'est avéré utile pour cerner et analyser quelques différences entre les deux modèles de navigation professionnelle. Ce cadre pourrait éventuellement servir pour l'étude évaluative d'autres modèles de navigation (Huberman \& Miles, 1991; Mukamurera, Lacourse \& Couturier, 2006).

\section{Implications}

La navigation professionnelle offre des réponses à certains enjeux liés à la continuité des soins tout en favorisant l'empowerment des patients et celui des familles. Les résultats appuient et illustrent la nature bidimensionnelle de ce rôle dans deux provinces. Ils semblent également indiquer que ce rôle doit être mieux défini pour être compris par les membres de l'équipe d'oncologie et par les IP ellesmêmes. Ce rôle n'est pas un simple remède ponctuel, permettant de pallier à ce ce qui fonctionne mal dans un contexte donné. En fait, la description des obstacles dans la continuité des soins dispensés aux patients-comme l'absence de mécanismes de transfert systématiques et de dossiers électroniques, les limites au niveau des outils, le manque d'intégration au sein des équipes d'oncologie officielles, les contraintes en matière d'espace physique et l'accès réduit aux IP en oncologie attribuable à la charge professionnelle croissante-permet de mieux comprendre ce qui fonctionne mal et la façon de remédier à la situation. L'adoption d'un cadre de navigation par les décideurs, les gestionnaires et les cliniciens pourrait mener à la création de rôles semblables pour d'autres maladies chroniques comme le diabète ou les affections cardiaques. De futures études pourraient examiner l'implantation de la navigation dans d'autres contextes et pour d'autres populations.

\section{Conclusion}

Les différences observées entre les deux modèles ou applications de navigation professionnelle s'expliquent en grande partie par des différences dans les milieux de travail. De façon générale, les deux modèles comportent des fonctions et des effets similaires. Les IP peuvent tout aussi bien être intégrées au sein d'une équipe spécialisée en oncologie ou dans la communauté. Toutefois, peu importe le milieu, elles doivent faire partie d'un réseau intégré de soins et de services au sein duquel le patient occupe la place centrale. Cela pourrait contribuer à améliorer la qualité et la continuité des soins en cancérologie. 


\section{RÉFÉRENCES}

Association canadienne des infirmières en oncologie. (2006). Normes de pratique et compétences pour l'infirmière spécialisée en oncologie. Récupéré de http://www.cano-acio.ca/conep/

Bowman, S.C. \& Grim, R.D. (2008). The efficacy of the nurse navigator in a community hospital breast care program. Seminars in Breast Disease, 11(1), 26-30. doi:10.1053/j.sembd.2008.04.007

Bulsara, C., Styles, I., Ward, A.M. \& Bulsara, M. (2006). The psychometrics of developing the patient empowerment scale. Journal of Psychosocial Oncology, 24(2), 1-16. doi:10.1300/ J077v24n02_01

Cancer Care Nova Scotia. (2004, March). Cancer patient navigation evaluation findings. March final report. Retrieved from http:// www.cancercare.ns.ca/en/home/publications/reports.aspx

Carver, C.S., Scheier, M.F., \& Weintraub, J.K. (1989). Assessing coping strategies: A theoretically based approach. Journal of Personality and Social Psychology, 56(2), 267-283. Retrieved from http://www. psy.miami.edu/faculty/ccarver/documents/p89COPE.pdf

Cook, S., Fillion, L., Veillette, A.-M., Cook, S., De Serres, Doll, R., \& Kazanjian, A. (2010, May). Scope of practice and associated competency requirements for professional cancer patient navigators. Symposium conducted at the IPOS 12th World Congress of Psycho-Oncology, Quebec City, Quebec, Canada/ Psycho-Oncology, 19(Suppl. S2), S12.

de Serres, M., \& Beauchesne, N. (2000). L'intervenant pivot en oncologie, un rôle d'évaluation, d'information et de soutien pour le mieux-être des personnes atteintes de cancer. Récupéré de http:// publications.msss.gouv.qc.ca/acrobat/f/documentation/2000/00908.pdf

Direction de la lutte contre le cancer (DLCC). (2007). L'infirmière pivot en oncologie. Programme de formation. Récupéré de http://www. msss.gouv.qc.ca/sujets/prob_sante/cancer/download.php?f=f3ca 389ceab25385666e2f00e2af485f

Doll, R., Barroetavena, M.C., Ellwood, A.L., Fillion, L., Habra, M.E., Linden, W., \& Stephen, J. (2007). The cancer care navigator: Toward a conceptual framework for a new role in oncology. Oncology Exchange, 6(4), 28-35. Retrieved from http://www.oncologyex. com/gif/archive/2007/vol6_no4/6_continuing_care_4.pdf

Earle, C.C. (2006). Failing to plan is planning to fail: Improving the quality of care with survivorship care plans. Journal of Clinical Oncology, 24(32), 5112-5116. doi:10.1200/JCO.2006.06.5284

Fillion, L., Cook, S., Blais, M.-C., Veillette, A.-M., Aubin, M., de Serres, M., ... Fournier, B. (2011). Implementation of screening for distress with professional cancer navigators. Oncologie, 13, 277-289. doi:10.1007/s10269-011-2026-8

Fillion, L., Cook, S., Veillette, A.-M., de Serres, M., Aubin, M., Rainville, F., ... Doll, R. (2012). Professional navigation framework: Elaboration and validation in a Canadian context. Oncology Nursing Forum, 39(1), E58-E69. doi:10.1188/12.ONF.E58-E69

Fillion, L., de Serres, M., Lapointe-Goupil, R., Bairati, I., Gagnon, P., Deschamps, M., ... Demers, G. (2006). Implantation d'une infirmière pivot en oncologie dans un centre hospitalier universitaire. Revue canadienne de soins infirmiers en oncologie, 16(1), 5-10.

Fitch, M.I. (2008). Supportive care framework: Theoretical underpinnings. In M.I. Fitch, H.B. Porter, \& B.D. Page (Eds.), Supportive care framework: A foundation for person-centered care (pp. 11-29). Pembroke, Ontario: Pappin Communications.

Haggerty, J.L., Reid, R.J., Freeman, G.K., Starfield, B.H., Adair, C.E., \& McKendry, R. (2003). Continuity of care: A multidisciplinary review. BMJ, 327(7425), 1219-1221. doi:10.1136/bmj.327.7425.1219

Huberman, M., \& Miles, M.B. (1991). Analyse des données qualitatives: Recueil de nouvelles méthodes [Qualitative data analysis: A sourcebook of new methods]. Bruxelles, Belgium: De Boeck Université.
Institute of Medicine (IOM). (2006). From Cancer Patient to Cancer Survivor: Lost in Transition. Retrieved from http://www.nap.edu/ openbook.php?isbn=0309095956

Lantz, P.M., Keeton, K., Romano, L., \& Degroff, A. (2004). Case management in public health screening programs: The experience of the national breast and cervical cancer early detection program. Journal of Public Health Management and Practice, 10(6), 545-555.

Mukamurera, J., Lacourse, F. \& Couturier, Y. (2006). Des avancées en analyse qualitative: Pour une transparence et une systématisation des pratiques. Recherches Qualitatives, 26(1), 110-138. Récupéré de http://www.recherche-qualitative.qc.ca/numero26(1)/mukamurera_ al_ch.pdf

Parker, V.A., Clark, J.A., Leyson, J., Calhoun, E., Carroll, J.K., Freund, K.M., \& Battaglia, T.A. (2010). Patient navigation: Development of a protocol for describing what navigators do. Health Services Research, 45(2), 514-531. doi:10.1111/j.1475-6773.2009.01079.x

Partenariat canadien contre le cancer (2010, janvier). L'expérience globale du cancer - Guide d'implantation de programmes de navigation en oncologie [Document de travail]. Récupéré de http://www.partnershipagainstcancer.ca/wp-content/uploads/FRNavigation-Guide_080910-FINAL-_2_.pdf

Partenariat canadien contre le cancer (2009, 21 mai). Guide d'implantation du dépistage de la détresse, le $6^{\text {ième }}$ signe vital. Vers des soins centrés sur la personne. Partie A : Contexte, recommandations et implantation [Document de travail]. Récupéré de http://www.partnershipagainstcancer.ca/wp-content/uploads/ 2.4.0.1.4.5-Guide_CJAG_FR.pdf

Patton M.Q. (1997). Utilization-focused evaluation: The new century text (3rd ed.). Thousand Oaks, CA: Sage Publications.

Plante, A., \& Joannette, S. (2009a). L'intégration des infirmières pivots dans les équipes d'oncologie en Montérégie : un aspect de l'implémentation du Programme de lutte contre le cancer, Partie 1. Revue canadienne de soins infirmiers en oncologie, 19(1), 13-18.

Plante, A., \& Joannette, S. (2009b). Centre intégré de cancérologie de la Montérégie : L'intégration des infirmières pivots dans les équipes d'oncologie en Montérégie : le processus. Partie 2. Revue canadienne de soins infirmiers en oncologie, 19(2), 72-77.

Richardson, A., Sitzia, J., Brown, V., Medina, J., \& Richardson, A. (2005). Patients' needs assessment tools in cancer care: Principles and practice. London: King's College London.

Skrutkowski, M., Saucier, A., Eades, M., Swidzinski M., Ritchie, J., Marchionni, C., \& Ladouceur, M. (2008). Impact of a pivot nurse in oncology on patients with lung or breast cancer: Symptom distress, fatigue, quality of life, and use of healthcare resources. Oncology Nursing Forum, 35(6), 948-954. doi:10.1188/08.ONF.948-954

Strauss A.L., \& Corbin J.M. (1990). Basics in qualitative research: Grounded theory procedures and techniques. Newbury Park, CA: Sage Publications.

Thorne, S., \& Truant, T. (2010). Les intervenants pivots solutionnerontils le problème? Les soins infirmiers en oncologie en transition. Revue canadienne de soins infirmiers en oncologie, 20(3), 122-128.

Tremblay, D. (2008). La traduction d'une innovation organisationnelle dans les pratiques professionnelles de réseau : L'infirmière pivot en oncologie (Dissertation doctorale, Université de Montréal, Québec, Canada). Récupéré de http://proquest.umi.com/ pqdlink?Ver=1\&Exp=10-19-2015\&FMT=7\&DID=1868855981\&RQT $=309 \&$ attempt $=1 \& \mathrm{cfc}=1$

Wen, K.Y., \& Gustafson, D.H. (2004). Needs assessment for cancer patients and their families. Health and Quality of Life Outcomes, 2, 11. doi:10.1186/1477-7525-2-11

Wilcox, B., \& Bruce, S.D. (2010). Patient navigation: A «win-win» for all involved. Oncology Nursing Forum, 37(1), 21-25. doi:10.1188/10. ONF.21-25

Yin, R.K. (1994). Case study research. Design and methods (2nd ed.). London: Sage Publications. 
Annexe 1: Guide de discussion-focus groupe

\section{FOCUS GROUPE}

\section{Guide de discussion}

Pour un groupe de six à huit participants, dont des IPC et d'autres professionnels qui travaillent avec les IPC

- Présentation de l'animatrice et des assistants

- Introduction de la recherche

- Justification du choix des participants et tour de table

- Introduction des participants.

Démarche: la discussion va porter sur les 3 grands thèmes suivants :

1. Pourquoi et comment le rôle des IPOs a-t-il été implanté?

2. Les principales fonctions du rôle des IPOs

3. Les effets et les impacts de la présence des IPOs

Durée : la discussion durera au maximum 2 heures.

Consignes.

Formulaire de consentement: lecture et signature.

Commencer avec la technique d'association de mots.

Distribution des stylos. Je vous demanderais d'écrire, à l'endos de votre formulaire de consentement, tous les mots qui vous viennent spontanément en tête, lorsque je vous entendez le terme : « infirmière pivot en oncologie ».

1. Pourquoi et comment?

Récemment, un nouveau rôle a été créé, celui des infirmières pivots en oncologie (IPOs) en CH-CSSS.

Selon vous

- Pourquoi ce nouveau rôle a-t-il été implanté au sein de votre organisation?

- Qui est responsable de l'implantation de ce nouveau rôle?

- Quels sont les objectifs visés en créant et en implantant ce nouveau rôle en oncologie?

- Selon-vous, quelle est la clientèle cible des IPOs?

- Quels sont les principaux problèmes rencontrés par ces personnes?

- Pourquoi a-t-on choisi de créer le rôle des IPOs pour y répondre?

- Selon vous, ce rôle est-il approprié pour répondre à ces principaux problèmes?

- Quand et comment a eu lieu l'implantation de ce nouveau rôle dans votre organisation?

- Quel type de préparation a été réalisé avant l'implantation de ce nouveau rôle?

- Selon vous, est-ce que les gens de votre organisation croyaient à la pertinence et à l'utilité de ce rôle dès le départ?

- Maintenant, comment ce rôle est-il perçu?

- Que pensez-vous de la pertinence de ce rôle?

\section{Les principales fonctions du rôle des IPOs}

Selon vous, quelles sont les principales fonctions de l'IPO?

FONCTIONS CLINIQUES, EMPOWERMENT (Centrées sur les besoins des patients)

- De quelle manière l'IPO contribue-t-elle à l'empowerment des patients? Quels outils sont utilisés?

- ÉVALUATION - DÉPISTAGE - TRIAGE - RÉFÉRENCE (Comment)

- SOUTIEN, SUPPORT ÉMOTIONNEL (Comment)

- INFORMATION (Comment)

- AUTRES (Gestion de symptômes, référence)

FONCTIONS DE COORDINATION, CONTINUITÉ DES SOINS

- Est-ce que l'IPO contribue à l'amélioration de la continuité des soins?

- Continuité de l'INFORMATION (Information globale sur le patient: bio-psycho-sociale et spirituelle + circulation de l'information)

- Continuité des TRAITEMENTS, des APPROCHES MÉDICALES (Coordination des services, traitements et suivis médicaux, trajectoire de soins intégrés)

- Continuité de la RELATION (IPO facilement accessible par le patient et ses proches tout au long de sa trajectoire de soins)

Selon vous, la population ciblée est-elle rejointe? Comment?

- Quand dans la trajectoire de soins sont-ils référés à l'IPO?

(Diagnostic - Début traitement - Pendant traitement - Fin traitement - Après traitement)

- Comment s'établit le contact entre cette clientèle et l'IPO? Est-ce qu'elle les contacte? Où est-elle basée? Est-ce qu'elle fait des activités à domicile? Quels sont les moyens de communication utilisés avec les patients?

Il y a 2 transitions critiques dans la trajectoire de soins des patients. Quelles sont les principales actions de l'IPO

- Autour du diagnostic?

- Et à la fin des traitements? 
Quelles sont les ressources utilisées par les IPOs facilitant leurs fonctions cliniques?

- Outils de référence, outils d'évaluation, documentation, accès aux dossiers

- Lorsque l'IPO a une difficulté, quels mécanismes sont mis à sa disposition? (Fonctions cliniques, fonctions de coordination)

Selon vous, qui sont les principaux partenaires des IPOs et comment collaborent-ils ensemble?

- Est-ce que les IPOs des équipes locales communiquent avec les IPOs des équipes régionales ou suprarégionales (IPOs spécialisées sur un site de cancer)? Comment?

- Est-ce que les IPOs communiquent avec les médecins de famille? Comment?

- Est-ce que les IPOs communiquent avec d'autres ressources? (organisations communautaires)

Qu'est ce qui facilite le rôle de l'IPO?

Qu'est ce qui rend son rôle difficile? (Avoir à faire des tâches qui ne lui appartiennent pas, manque de ressources)

3. Effets ou impacts

Selon vous, quelles sont les conséquences reliées à la présence de ce rôle pour les personnes atteintes et leurs proches?

- Pour les patients et leurs proches

- Soutien, support émotionnel

- Information et autres besoins

- Accès aux services, ressources dans une courte durée de temps

- Préparation pour les prochaines étapes de la trajectoire

- Satisfaction et qualité de vie

Quelles sont les conséquences de la présence de ce rôle sur le système de la santé?

- Pour la continuité des soins

- Circulation de l'information relative au patient (de façon à ce que le patient n'ait pas à répéter son histoire encore et encore)

- Communication entre les établissements, notamment entre l'équipe spécialisée et la première ligne

- Communication entre les professionnels de la santé (collaboration interprofessionnelle)

- Communication entre les médecins de famille et l'équipe specialise

- Communication entre les médecins de famille et la personne atteinte

À plus long terme, quels pourraient être les effets ou impacts de la présence de ce rôle sur le système de santé? (Souhaités ou non)

Fin de l'entrevue - Merci $:$

Annexe 2: Guide d'entrevue individuelle

\section{GUIDE D'ENTREVUE INDIVIDUELLE - Intervenants}

1. Les origines

Récemment, un nouveau rôle a été créé, celui des infirmières pivots en oncologie (IPOs) en CH-CSSS. Selon vous, pourquoi ce nouveau rôle a-t-il été créé au sein de votre organisation?

- Quelles sont les origines de ce nouveau rôle?

- Qui est responsable de l'implantation de ce nouveau rôle?

- Quels sont les objectifs visés en créant et en implantant ce nouveau rôle en oncologie?

- Selon-vous, quelle est la clientèle cible des IPOs?

- Quels sont les principaux problèmes rencontrés par ces personnes?

- Pourquoi a-t-on choisi de créer le rôle des IPOs pour y répondre?

- Selon vous, ce rôle est-il approprié pour répondre à ces principaux problèmes?

- Quand a eu lieu l'implantation de ce nouveau rôle dans votre organisation?

- Quel type de préparation a été réalisé avant l'implantation de ce nouveau rôle?

- Quand avez-vous été informé de l'implantation de ce nouveau rôle?

- Comment en avez-vous été informé?

- Estimez-vous avoir reçu assez d'informations à ce sujet? Si oui, qu'avez-vous apprécié comme mode d'information? Si non, qu'auriez vous aimé recevoir comme information et comment?

- Selon vous, est-ce que les gens de votre organisation croyaient à la pertinence et à l'utilité de ce rôle dès le départ?

- Maintenant, comment ce rôle est-il perçu?

- Que pensez-vous de la pertinence de ce rôle?

2. Les activités

Selon vous, quelles sont les principales fonctions de l'IPO?

FONCTIONS CLINIQUES

- Contribue-t-elle à rendre le patient et ses proches plus aptes à répondre à leurs besoins occasionnés par cette maladie? Comment?

ÉVALUATION - DÉPISTAGE - TRIAGE - RÉFÉRENCE

- Est-ce que l'IPO évalue les besoins de la personne atteinte et de ses proches?

- Quand le fait-elle? Lors du diagnostic? A la fin des traitements?

- Utilise-t-elle des outils particuliers lors de l'évaluation?

- Qui peut accéder à ces informations? Comment?

- Est-ce utile? Pourrait-il y avoir amélioration?

suite à la page 276 .. 
SOUTIEN

- Est-ce que l'IPO joue un rôle de soutien auprès la personne? Comment?

- Quel type de soutien donne-t-elle? (soutien émotionnel, pratique ou instrumental pour résoudre des problèmes concrets, soutien spirituel, autre type de soutien) Comment?

- Quelles sont les habiletés que doit posséder une IPO pour bien soutenir la personne atteinte et ses proches? (communication)

- L'IPO est-elle soutenue par une équipe en oncologie psychosociale et spirituelle?

- Comment l'IPO collabore-t-elle avec les autres professionnels de l'équipe d'oncologie psychosociale?

INFORMATION

- Est-ce que l'IPO donne de l'information à la personne atteinte et à ses proches? Quelle sorte d'information? Comment?

AUTRES FONCTIONS CLINIQUES

- L'IPO accomplit-elle d'autres fonctions cliniques? (Gestion de symptômes, référence)

- Est-ce que le temps que l'IPO consacre à l'accomplissement de ces autres fonctions cliniques est une partie importante de sa pratique? A-t-elle assez de temps pour accomplir ces autres fonctions?

\section{FONCTIONS DE COORDINATION, CONTINUITÉ DES SOINS}

- Est-ce que l'IPO contribue à l'amélioration de la continuité des soins? Comment? (Coordination des soins)

- Continuité de l'information. Est-ce que l'IPO contribue à ce que l'information circule, à ce que le patient n'ait pas à répéter son histoire encore et encore? Comment?

- Continuité des traitements, des approches médicales. Est-ce que l'IPO facilite la coordination des services? (traitements et suivis médicaux, continuité des soins) Comment?

- Continuité de la relation. Est-ce que l'IPO est facilement accessible pour le patient et ses proches tout au long de sa trajectoire de soins?

Est-ce qu'une, ou plusieurs de ces fonctions, semble prédominante?

Est-ce l'IPO se retrouve à faire des tâches qui ne lui appartiennent pas?

Qu'est ce qui facilite le rôle de l'IPO?

Qu'est ce qui rend son rôle difficile?

\section{La participation}

Selon vous, la population ciblée est-elle rejointe?

- Comment s'établit le contact entre cette clientèle et l'IPO? (moyens de communication)

- Comment les patients sont-ils référés à l'IPO?

- Quand dans la trajectoire de soins sont-ils référés à l'IPO?

(Diagnostic - Début traitement - Pendant traitement - Fin traitement - Après traitement)

- Il y a 2 transitions critiques dans la trajectoire de soins des patients. Quelles sont les principales actions de l'IPO autour du diagnostic? Et à la fin des traitements?

Comment l'IPO travaille avec les autres professionnelles de la santé ou les membres de la communauté?

- Où l'IPO est-elle basée?

- Fait-elle des activités à domicile?

- Est-ce que l'IPO est intégrée au sein d'une équipe d'oncologie? Comment?

- Est-ce que des actions ont été entreprises afin de faciliter son intégration au sein de l'équipe? Lesquelles?

- Avez-vous eu à contacter ou à rencontrer une IPO?

- Vous a-t-elle contactée? De quelle façon?

- Selon vous, qui sont les principaux partenaires des IPOs?

- Comment collaborent-ils ensemble?

- Quels sont les autres intervenants qui ont accès aux IPOs? (Travailleurs sociaux, psychologues, pharmaciens)

- Comment ceux-ci ont-ils accès aux IPOs?

- Est-ce que les IPOs des équipes locales communiquent avec les IPOs des équipes régionales ou suprarégionales (IPOs spécialisées sur un site de cancer)? Comment?

- Qu'est ce qui facilite cette communication?

- Qu'est ce qui la rend difficile?

- Est-ce que les IPOs communiquent avec les médecins de famille? Comment?

- Qu'est ce qui facilite cette communication?

- Qu'est ce qui la rend difficile?

- Est-ce que les IPOs communiquent avec d'autres ressources? (org. communautaires)

Comment l'IPO utilise les ressources mises à sa disposition?

- Quelles sont les ressources utilisées par les IPOs facilitant leurs fonctions cliniques? (Outils de référence, outils d'évaluation, documentation, accès aux dossiers)

- Est-ce que ces ressources sont suffisantes?

- Lorsque l'IPO a une difficulté, quels mécanismes sont mis à sa disposition?

- Est-elle soutenue dans ces fonctions cliniques? À qui peut-elle se référer?

- Est-elle soutenue dans ces fonctions de coordination? À qui peut-elle se référer?

- A votre avis, est-ce que les IPOs peuvent adapter les services qu'elles offrent en fonction des besoins de la personne atteinte? 


\section{Les réactions}

Que pensez-vous de la pertinence du rôle de l'IPO pour...?

- La personne atteinte et ses proches

- Les médecins et les intervenants du CH

- Le médecin de famille des personnes atteintes

- Est-ce que ce rôle est utile à d'autres personne?

\section{Les effets sur les personnes atteintes de cancer}

Selon vous, quelles sont les conséquences reliées à la présence de ce rôle pour les personnes atteintes et leurs proches?

- Selon vous, est-ce que la personne et ses proches se sentent mieux équipés pour faire face à la maladie grâce à la présence de l'IPO? (Empowerment)

- Comment? (Capacité de résolution de problèmes, coping actif, perception d'efficacité personnelle, détresse, bien-être, qualité de vie)

- Est-ce que les acteurs du réseau perçoivent que la personne atteinte et ses proches arrivent mieux préparés lors d'épisodes de traitement ou de suivi depuis l'implantation des IPOs?

Est-ce que la présence de l'IPO a d'autres effets positifs sur la personne atteinte et ses proches?

Est-ce que la présence de l'IPO peut avoir des effets négatifs ou non souhaités sur la personne atteinte et ses proches? Si oui, lesquels?

6. Les effets sur le système de la santé et la continuité des soins

Quelles sont les conséquences de la présence de ce rôle sur le système de la santé?

- Est-ce que l'information à propos du patient circule mieux au sein de l'organisation de façon à ce que le patient n'ait pas à répéter son histoire encore et encore?

- Est-ce que l'IPO facilite le travail en interdisciplinarité parmi les professionnels de la santé gravitant autour des patients? Comment?

- Est-ce que l'IPO contribue à ce que l'information circule mieux entre les établissements, notamment entre l'équipe spécialisée et la première ligne?

- Est-ce que la présence d'une IPO facilite la relation ou la communication :

- Entre les professionnels de la santé? (Collaboration interprofessionnelle)

- Entre le médecin de famille et l'équipe spécialisée?

- Entre le médecin de famille et la personne atteinte?

- Est-ce que l'IPO contribue à améliorer la coordination des soins?

- Est ce que la personne atteinte a une meilleure compréhension de ce système de soins complexe?

- A-t-elle ainsi un meilleur accès aux soins de support et à d'autres services?

- Est-ce que cela contribue à une meilleure compréhension de la trajectoire de soins?

- Est-ce que l'IPO permet d'augmenter la satisfaction face à la qualité des services et des soins offerts?

- Est-ce que la présence de l'IPO contribue au maintien d'une continuité relationnelle pour le patient? Comment?

- Est-ce l'IPO devient une personne de référence pour le patient et ses proches? (filet de sécurité)

Y a-t-il d'autres effets positifs liés à la présence de ce rôle sur le système de santé?

Y a-t-il des effets négatifs ou non souhaités à la présence de ce rôle sur le système de santé?

\section{Autres effets ou impacts à plus long terme}

À plus long terme, quels pourraient être les effets ou impacts de la présence de ce rôle sur le système de santé? (Souhaités ou non)

\section{Autres commentaires}

Fin de l'entrevue - Merci $\odot$ 